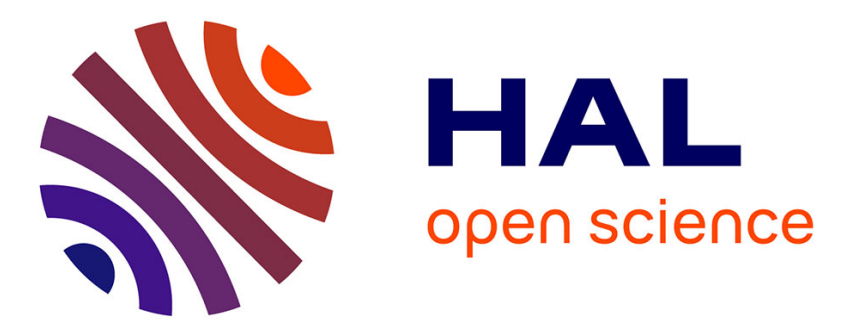

\title{
Intuitive Human Robot Interaction based on unintentional synchrony: a psycho-experimental study
}

Syed Khursheed Hasnain, Ghiles Mostafaoui, Robin Salesse, Ludovic Marin, Philippe Gaussier

\section{- To cite this version:}

Syed Khursheed Hasnain, Ghiles Mostafaoui, Robin Salesse, Ludovic Marin, Philippe Gaussier. Intuitive Human Robot Interaction based on unintentional synchrony: a psycho-experimental study. IEEE Joint conference on Development and learning and on Epigenetic Robotics, Aug 2013, Osaka, Japan. hal-00834549

\author{
HAL Id: hal-00834549 \\ https://hal.science/hal-00834549
}

Submitted on 16 Sep 2013

HAL is a multi-disciplinary open access archive for the deposit and dissemination of scientific research documents, whether they are published or not. The documents may come from teaching and research institutions in France or abroad, or from public or private research centers.
L'archive ouverte pluridisciplinaire HAL, est destinée au dépôt et à la diffusion de documents scientifiques de niveau recherche, publiés ou non, émanant des établissements d'enseignement et de recherche français ou étrangers, des laboratoires publics ou privés. 


\title{
Intuitive Human Robot Interaction based on unintentional synchrony: a psycho-experimental study
}

\author{
Syed Khursheed Hasnain ${ }^{1}$, Ghiles Mostafaoui ${ }^{1}$, Robin Salesse ${ }^{2}$, Ludovic Marin ${ }^{2}$,Philippe Gaussier ${ }^{1}$ \\ ${ }^{1}$ Neurocybernetic team, ETIS ENSEA, University of Cergy-Pontoise 95302 France. \\ Email:syed-khursheed.hasnain, ghiles.mostafaoui, gaussier at ensea.fr \\ ${ }^{2}$ Movement to Health Laboratory, EuroMov, Montpellier-1 University Montpellier, France \\ Email:robin.salesse, ludovic.marin, gaussier at@univ-montp1.fr
}

\begin{abstract}
Inspired by studies of interpersonal coordinations, we assumed that unintentional synchrony is a fundamental parameter to initiate and maintain Human Machine interactions. We developed a neural model allowing a robot to synchronize its behavior depending on human movement frequency, and thus to choose this partner on the basis of synchrony detection between its own learned dynamic and the visual stimuli induced by the human. To confirm or deny our assumptions we presented here a psychological study to measure unintentional synchronization during Unidirectional and Bidirectional Human Robot Interaction using our previously proposed model for initiating the interaction and focusing the robot attention on a selected partner. The experimental results demonstrated that bidirectional intuitive interaction leading to possible unintentional synchronization is primordial to obtain natural human robot interactions using minimal cognitive load (unintentional behavior).
\end{abstract}

\section{INTRODUCTION}

As robots start moving closer to our social and daily lives, issues of agency, sociality and believability become more important. However, despite noticeable advances in Human Robot Interaction (HRI), the developed technologies induce two major drawbacks: (i) HRI are highly demanding, (ii) humans have to adapt their way of thinking to the potential and limitations of the Robot. Thereby, HRI induce an important cognitive load which question the acceptability of the future robot. Consequently, we can address the question of understanding and mastering the development of pleasant yet efficient human robot interactions which increase self- esteem, engagement (or pleasure), and efficacy of the human when interacting with the machine.

In this race for more user-friendly HRI systems (robotic companion, intelligent objects etc.), working on the technical features (the design of appearance and superficial traits of behavior) can contribute to some partial solutions for punctual or short- term interactions. For instance, a major focus of interest has been put on the expressiveness and the appearance of robots and avatars [1] [2][3]. Yet, theses approaches have neglected the importance of understanding the dynamics of the interactions.

In our opinion, intuitive communication refers to the ability of the robot to detect the crucial signals of the interaction and use them to adapt one's dynamics to the other's behavior.
In fact, this central issue is highly dependent on the robot's capacities to sense the human world and interact with it in ways that emulate collaborative human-human work.

In early communication among humans, synchrony was found to be a fundamental mechanism relying on very lowlevel sensory-motor networks, inducing the synchronization of inter- individual neural populations from sensory flows (vision, audition, or touch) [4][5]. Synchrony is caused by the interaction but also sustains the interaction itself in a circular way, as promoted by the enaction approach [6]. Consequently, to become a partner in a working together scenario, the machine can obtain a minimal level of autonomy and adaptation by predicting the rhythmic structure of the interaction to build reinforcement signals to adapt the robot behavior as it can maintain the interest of the human in more long-term interactions.

More precisely, as we are aiming for a more "intuitive" and "natural" HRI, we took advantages of recent discoveries in low-level human interactions and studied Unintentional Synchronizations during rhythmic human robot interactions. We argue that exploiting natural stability and adaptability properties of unintentional synchronizations and rhythmic activities can solve several of the acceptability problems of HRIs, and allow rethinking the current approaches to design them.

Based on these assumptions, we proposed in our recent works a neural model permitting to a NAO robot to initiate an interaction with a selected human partner and to locate its focus of attention on this preferred interactant on the basis of synchrony detection [7]. A summarized description of this model is given section III.

In order to confirm or deny our assumptions on the influence of unintentional synchronizations for intuitive HRI, we propose in this article a collaborative psychological study to analyze unintentional synchronizations during rhythmic interactions involving a NAO robot and naive human partners. Our objective is to measure the quality of unintentional synchronizations during the interactions in two different scenarios : (i) with NAO moving with a fixed frequency of interaction (Unidirectional interaction) (ii) with NAO having minimal abilities (by using our synchrony based neural model) to adopt the human partner frequency of interaction (Bidirectional interaction). 


\section{THEORETICAL CONTEXT}

Synchrony is ubiquitous in nature and appear to be an important parameter for communication for most of the living species. As good examples we can mention flashing of fireflies [8], cricket chirping [9], circadian rhythm [10], birds in flocks synchronizing takeoff and landing [11] and male and female mosquitoes synchronizing wing beats [12].

Social psychology acknowledged synchrony as an essential integrated part of human communication [13]. For instance human verbal interaction is not only speech dependent, in fact, many non-verbal behaviors such as facial expressions, hand movements and some other gestures are also to be considered as important parameters to facilitate the interaction. These behaviors are synchronized naturally during human social interactions [14]. Moreover, the ability to synchronize with each other is tightly linked to the quality of the communication or interaction [15].

From a neurobiological point of view, several studies used fMRI and EEG to record the brain activities during social interaction. Stephens et al. recorded the brain activities of a speaker (reciting a monologue) and then scanned the brain of a listener, it was found that there is a temporal and spatial coupling between the listener and the speaker [16]. Recently, a new technique called hyperscanning (used with fMRI and EEG) made possible to record the brain activities of two persons simultaneously when they are engaged in social interaction. Babiloni et al. observed that, during card playing if one of the participants have to play a card (as a response to the play of the other), a brain activity is visible in the right prefrontal and parietal areas of both the first player and the companion [17]. Recent works of Dumas et al. [18] using hyperscanning has revealed the emergence of inter- brain synchronization across multiple frequency bands during social interactions (spontaneous exchanges between two participants of intransitive bi-manual movements). Examining the phase synchronization between the two brains, it appears that these synchronous exchanges exhibit the emergence of an interindividual brain- web (linked to the sensorimotor information) across several frequency bands. Symmetrical patterns were found in low frequency band (may be due to coordinated dynamics of hand movements) and asymmetric in higher frequency. From all these data, we can conclude that these inter subject synchronizations are not planned as high level processing but result in low level analogical synchronization of neural populations from the sensory flow (vision, audition etc.) otherwise millisecond synchronization would never be obtained.

One of the interesting aspects of these social synchronizations is their Unintentional nature. In fact, synchrony or entrainment is also used as a physiological indexe (for instance heart rate variability and respiration [19]). This physiological entrainment could be considered as an important way of communication among interactants through mutual bodies [20]. Watanabe et al. showed the existence of respiratory entrainment among interactants during face to face communication [21]. Ruth Feldman demonstrated the powerful impact of maternal-infant social contact on the infant physiological systems, his bootstrapping analysis indicates that mother and infant coordinate heart rhythms through episodes of interactional synchrony [22].
Studies of Interpersonal motor coordination point out this very low level mechanism of unintentional synchronization or communication among people. For example when an adult and child walk together, unconsciously they try to maintain the same phase by constantly adjusting the length or the frequency of their steps [23]. Moreover, individuals have their own clapping frequencies, but people tend to applaud in a synchronized way [24].

In addition, Issartel et al. studied interpersonal motorcoordination between two participants when they were instructed not to coordinate their movements. The results showed that participants could not avoid unintentional coordination with each other [23]. In recent research on schizophrenia, Varlet and al. examined intentional and unintentional social motor coordination in participants oscillating hand-held pendulums from the wrist. The results showed that for a participant suffering from schizophrenia, intentional motor coordination remained impaired however, unintentional social motor coordination was preserved. This study demonstrates that unconscious communication sustains even though the patients are suffering from social interaction disorder [25].

Furthermore, developmental psychology also acknowledges synchrony as a prime requirement for interaction between a mother and her infant [20]. An infant stops interacting with his mother when she stops synchronizing with him [26]. An interesting aspect of these inter- individual synchronizations is their unintentional nature (for instance infants synchronize their legs motion with adult speech [27]).

Mimicry is another good example of the influence of unintentional synchrony in the development process. For infants development, imitation has been suggested to play a key role in the learning of many abilities including motor skills and language as well as the understanding the goals, intentions, and desires of others [28]. Usually, imitation occurs when one person explicitly and intentionally performs anothe's motor movement like when a child imitates her mother's clapping. By contrast, mimicry is the unconscious tendency to perform another's behavior when interacting (i.e, the chameleon effect, [29] such as when one person's yawn causes another person to yawn. The matching of another's action by an observer is said to be mediated by the mirror neuron system located in the premotor and parietal brain regions that is activated when an action is both observed and performed [30] although the specific mechanisms responsible for intentional imitation and mimicry may be different [31]. Yet the intentional side of neonate imitation can be questioned and the differentiation between mimicry and imitation is perhaps an effect of the baby development.

Hence synchrony is a fundamental mechanism in early human communication. It provides a way to build reinforcement signal and to evaluate the quality of the interaction.

Different groups of researchers have started to investigate the stability of human-robot interactions using synchrony and anti-synchrony as a link between stable internal dynamics and bi-directional phase locking with the caregiver [32][33][34]. Blanchard and Canamero proposed a velocity detection system to synchronize the movements of two robots to improve the reactivity of agents to changes in their environment [35]. Michalowski et al. developed a dancing robot to analyses 


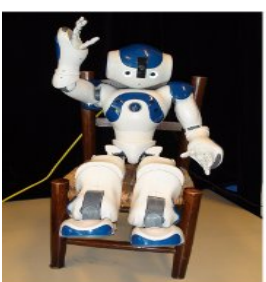

(a)

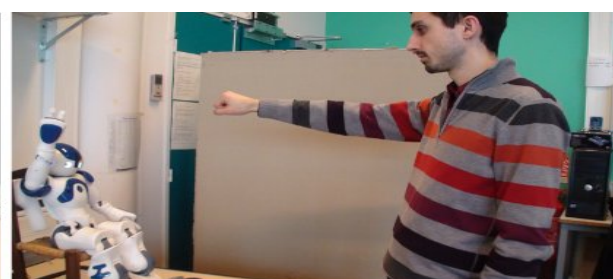

(b)
Fig. 1. Setup for our experiments. (a) Nao robot (b) human-robot interaction.

the properties and significance of synchronized movement in general social interaction [36]. Ikegami and Iizuka [37] used the genetic algorithm technique and showed that coupling and turn-taking between two agents are sensitive to the dynamics of interaction. Crick et al. programmed a robot for drumming (with human drummers) by integrating multiple sensors input (oscillators). They showed that precise synchronization between humans and robots can be achieved by fusing multiple sensors input although the incoming data is imperfect [38]. Moreover, Hafner and Kaplan presented the idea of interpersonal maps. These maps are the geometrical representation based on one's own behavior and the others. Using these maps, different types of interactions for instance imitation can be detected [39]. In the same way, we have shown that an expressive head can easily learn to recognize facial expression if the human caregiver resonate/imitate the robot facial expression. He/she provides a visual feedback correlated with the robot internal state. A classical online conditioning mechanism is then sufficient to learn the correct association [40][41].

Finally, Marin et al. underlined, in their psychological studies, that motor resonance between robots (humanoid) and humans could optimize the social competence of human-robot interactions [42].

\section{NEURAL MODEL FOR SYNCHRONY DETECTION AND PARTNER SELECTION}

The core of the presented work is a psychological investigation on unintentional synchronization during human robot rhythmic interactions. For the sake of clarity, before detailing this study, a summarized description of the neural model permitting NAO to interact with naive human subjects is given in this section. We invite the reader to refer to [7] for a complete picture of this model.

The original model developed in [7] is composed by three parts: a simple dynamical interaction model, a selection of partner architecture and a Focus of attention mechanism. However, here, we will use only the two first sub-parts of the model for initiating the interaction and selecting the partner on the basis of synchrony detection (see figure 2). The used experimental setup is illustrated in figure 1.

As a first step towards human-robot interactions, we use a simple dynamical interaction model (figure 2, part a) to provide minimal abilities to the robot to interact with humans on the basis of synchrony by adopting the phase and frequency of its partner. Figure 2 (part a, dotted box) shows the oscillator module controlling the robots dynamics (oscillations) [43]. It consists of two neurons $N 1$ and $N 2$ inhibiting each other proportionally to the variable $\beta$. The oscillating frequency is a function of the variables $\alpha 1, \alpha 2$ and $\beta$ :

$$
\begin{aligned}
& N_{1}(n+1)=N_{1}(n)-\beta N_{2}(n)+\alpha 1 \\
& N_{2}(n+1)=N_{1}(n)+\beta N_{2}(n)+\alpha 2
\end{aligned}
$$

Normally, the robots oscillator, representing the internal dynamic of the robot, oscillates at its own frequency and amplitude. Let now consider a human trying to interact with the robot by moving its arm in front of NAO. The motion in the visual field is then estimated by an optical flow algorithm [44], the velocity vectors are then converted into positive and negative activities. If the perceived movements are in the upward direction, the oscillator gets the positive activity and its amplitude increases on the positive side. On the contrary, if the negative activity is perceived, the amplitude goes down. Lets now rephrase the mathematical equation of the robot oscillator by:

$$
N_{1}(n+1)=N_{1}(n)-\beta N_{2}(n)+\alpha 1+f^{\prime}
$$

Where $f^{\prime}$ is the energy induced by the optical flow activities. Consequently, when an agent interacts, depending on the visual energy (optical flow) produced by its movements, the robots oscillator will be modified (frequency and phase) but within certain limits, defined by a coupling factor to avoid saturations.

This first model provides a basic architecture initiating automatically a human-robot interaction by synchronizing the agents movements (in an imitating framework). The Part (b) of Figure 2 describe a selection of partner model completing the previous one to choose an interacting partner among various interacting agents. It can be segregated into two parts. The first one is the dynamical interaction model (part a) and the other one is the frequency-prediction module. Previously, the robot's oscillator was directly linked to the external visual stimuli $f^{\prime}$ now, the coupling activities are linked with the frequencyprediction module $\left(f^{\prime \prime}\right)$. The equation of the oscillator 3 can be rephrase as : $N_{1}(n+1)=N_{1}(n)-\beta N_{2}(n)+\alpha 1+f^{\prime \prime}$

Where, $f^{\prime \prime}$ is the coupling energy feed by the frequencyprediction block. The other variables remain unchanged.

The selection of partner architecture works in two phases: a learning phase and a testing phase. During the learning phase, the robot perceives and learns its own dynamics. It initiates two processes. The frequency-prediction module starts now predicting the robots modifiable oscillator as a weighted sum of visual stimuli induced by its own movements. As a consequence, it also modifies the robots oscillator. This process of modifying, learning and adapting continues and converge after some time. We used a Least Mean Square (LMS) [45] algorithm for the learning of the frequency-prediction module.

After this learning phase, when an agent interacts with a frequency close to the learnt one, weights (that are already learnt on modifiable links of the LMS) are associated with the visual activities induced by the human movements and robots modifiable oscillator adopts the interactant frequency and phase. If the interacting frequency is different from the learnt one, the weights could not be associated with the visual 


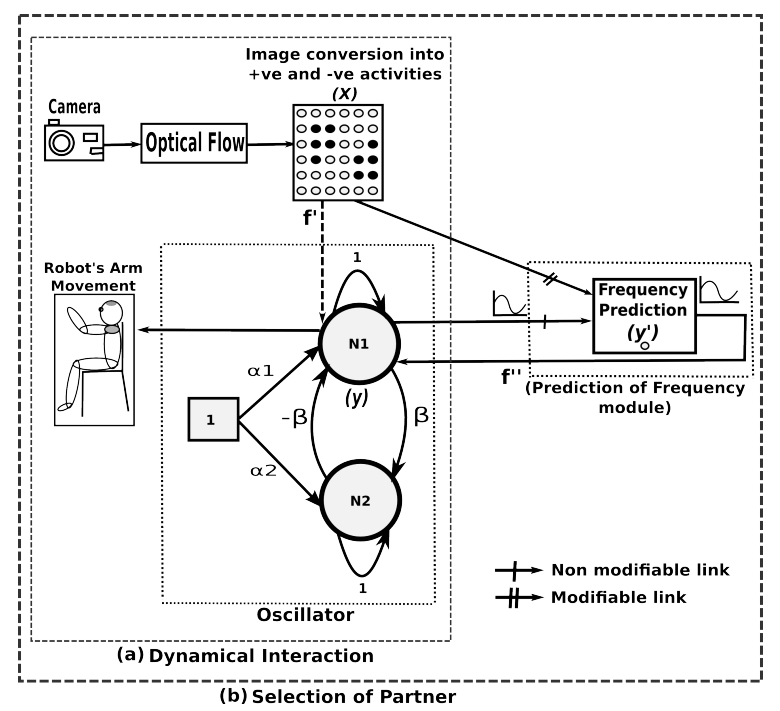

Fig. 2. (a) Dynamical Interaction model (b) Selection of Partner: select an interacting partner on the basis of synchrony detection among various interacting agents.

stimuli and the robot continues to move at its default frequency. The same is true in the case of multiple interacting agents. Among several interactants, only the agent having a similar dynamic (frequency) as the robot is selected. It is worth noticing that the range of accepted frequencies of interaction (around the learnt one) is dependent on the value of the coupling factor.

As it is described previously, Nao is able to select and interact with a partner having rhythmic movements similar or close to its inner dynamic represented by just one oscillator. One of the main goal behind this work is to use unintentional synchrony as an automatic starting point for human/robot interactions leading to a developmental learning of more complex tasks by imitation games. To do so, the robot must be able to select (synchronize) and interact with multiple agents having different dynamics of motion (or one agent moving with different complex movements). The robot must be capable of selecting partners in a larger band of frequencies (different dynamics of motion).

In order to test a possible generalization of our model to more complex tasks we introduced (using the same model described previously) three different oscillators $\mathrm{A}, \mathrm{B}$ and $\mathrm{C}$ with the following frequencies $f_{A}=0.441 \mathrm{~Hz}, f_{B}=0.83 \mathrm{~Hz}$ and $f_{C}=1.153 \mathrm{~Hz}$ respectively. All these oscillation frequencies are learnt by three different signal-prediction modules. These oscillators determine the Nao's current oscillation frequency and switch according to the visual stimulus. When there is no visual stimulus, one of the oscillators is randomly selected (every 4 seconds). This phase simulates in a very simple manner a babbling state (3 simple gestures randomly performed). As the interacting agent arrives and interacts with a given frequency, the signal-prediction module which is near to this frequency synchronizes with it. Our architecture selects among three oscillators, the one having the minimal error regarding to the external visual stimulus induced by the human partner. The reader can refer to [7] for more details about these experimental results.

\section{MATERIALS AND METHOD}

\section{A. Subjects}

In total, 15 young students from the university of Montpellier 1 ( 8 men, 7 women) participated in the experiments. Their ages vary between 18 to 25 years and their height between 1.56 $\mathrm{cm}$ to $1.9 \mathrm{~cm}$. Among the participants, 14 of them are right handed while a single one is left handed. All the subjects are healthy with no physical, psychological or neurophysiological impairments.

\section{B. Apparatus}

The experimental setup is similar to the one shown in the figure 1. Components of our experiment includes : Human naive subjects; a Nao robot with an added external camera to avoid the limitation of the Nao's camera which is limited to 10 $\mathrm{Hz}$ (frame rate) through the ethernet connection; a Laptop to control the robot; two Goniometers, a first one fixed on NAO's shoulder to measure its movements and a second one fixed on the subjects shoulder to measure their motion signals; another Laptop to records the Goniometers output signals.

The frame rate for our experiments is $30 \mathrm{~Hz}$. The distance between the human and the robot is about 1.5 meters. The external camera is set at the same distance from the subjects at a height of 0.9 meters from the ground. NAO's shoulder is at a distance of 1,3 meters from the ground.

\section{Conditions and Stimuli}

As introduced above, one of the main goal of this work is to study unintentional synchronizations for two different types of rhythmic human robot interactions : Unidirectional ones and Bidirectional ones.

Unidirectionality can be defined here as interactions where only one of the two agents (the human or the NAO robot) has the ability (or possibility) to synchronize his/its dynamics with the other partner who, in contrary, is moving with his/its own dynamics regardless to his/its external environment (blind conditions).

Therefore, Bidirectional interaction correspond to interactions involving agents (human and robot) with mutual capacities of synchronization.

Consequently, the experiments were performed using 4 different conditions, 2 unidirectional ones and 2 bidirectional ones. For all the conditions, no a priori knowledge was given to the subject regarding the way that the robot will move and react during the interaction. Each subject performed in a randomly chosen order 12 trials, 3 trials for each condition. Each trial lasted 30 seconds except those of the condition 4 which have durations of 60 seconds.

1) condition 1, Unidirectional, Blind and Deaf Human Vs NAO controlled by the simple synchrony model: The subjects are instructed to close their eyes while listening a white noise and to move their arm continuously and in oscillatory manner with their preferred frequency. In this condition, the subject are not able neither to perceive nor to hear NAO's movement. The reason behind this choice is to be sure that the subjects move with their "real" natural frequency by removing all the external visual or acoustic perturbations. 
Nao is controlled by the simple synchrony model. Its arm produce oscillatory movements with a frequency of 0.35 . The coupling factor is set to 0.4 giving the robots ability to adapt its motion dynamic to a large range of frequencies of interaction (approximatively from 0.2 to $0.5 \mathrm{~Hz}$ ).

This condition permits in particular to measure the natural motion frequency of each participant.

2) condition 2, Unidirectional, Blind NAO Vs human: As for the previous condition, the subjects are instructed to move their arm continuously and in an oscillatory manner with their preferred natural frequency while focusing their visual attention on NAO's arm.

Here, Nao is also controlled by the simple synchrony model but the camera was turned off. Consequently, NAO moves its arm with oscillatory movements corresponding to the first fixed frequency of $0,35 \mathrm{~Hz}$ but never adapt its movements to the human behavior (it can not perceive the external stimuli). NAO's arm can be seen in this condition as a pendulum oscillating with a fixed frequency.

3) condition 3, Bidirectional, Human Vs NAO controlled by the simple synchrony model: This condition is similar to the second one except the fact that NAO's camera was turned on. NAO's arm is then controlled in the same manner as in the condition 1 with capabilities to adapt its movements to the motion frequencies of the subjects. The large range of accepted frequencies of interaction is limited by the coupling factor which was set to 0.4 as in the condition 1 .

4) condition 4, Bidirectional, Human Vs NAO controlled by the partner selection model: The instruction for the subject remain the same as for the condition 2 and 3. NAO's arm is now controlled by the selection of partner model leading to abilities to synchronize its movements "only" with humans having motion frequencies close to one of these following learnt frequencies : $0,21 \mathrm{~Hz}, 0,35 \mathrm{~Hz}, 0,56 \mathrm{~Hz}$. The coupling factor is here set to a lower value of 0.07 , consequently Nao can synchronize only with small ranges of frequency which are approximatively : 0,19 to 0,22 for the first learnt frequency, 0,32 to 0,37 for the second one and finally 0,52 to 0,63 .

\section{Data Filtering and measurements}

We computed the shoulder angular displacements by reducting the 2 dimensional signals of the goniometers to a 1 dimensional signal using a Principal Component Analysis (PCA) on each trial and analysing only the first obtained componant. A fast Fourier transform (FFT) analysis performed on the data revealed that the frequency spectrum was essentially located below $1 \mathrm{~Hz}$. Thus, we filtered data with a second-order dualpass Butterworth filter with a $3 \mathrm{~Hz}$ cut-off frequency. Finally, linear trends have been removed from the obtained vectors. We used three measures to quantify and analyze synchrony from the obtained signals : the instantaneous frequency of both NAO and Humans as a function of time, the mean frequency over each trial of both NAO and Humans and an index of synchrony between NAO and the Human partner for each trial.

The instantaneous frequency $F i$ is the derivative function (in time) of the angle obtained after filtering the signals using Hilbert transform. The mean frequency $F m$ is the mean values of the instantaneous frequencies.
To obtain the index of synchrony $I s$ of two signals $S 1$ and $S 2$, we will use the following equation :

$I s=\frac{\left.\sum_{i=1}^{N} \cos \left(\theta_{h}^{S 1}(i)\right)-\theta_{h}^{S 2}(i)\right)}{N}+\frac{\left.\sum_{i=1}^{N} \sin \left(\theta_{h}^{S 1}(i)\right)-\theta_{h}^{S 2}(i)\right)}{N}$

$\theta_{h}^{S 1}$ and $\theta_{h}^{S 2}$ are respectively the Hilbert transform angles of the signals $S 1$ and $S 2$ after centering them relative to their mean value. $N$ is the number of samples.

\section{Results}

\section{A. condition 1 (Unidirectional)}

For this first condition, the subjects are in blind and deaf conditions, the main reason for that is to measure their natural and preferred motion frequency while all the possible external visual or acoustic disturbances are removed. We can notice that the human subjects frequencies varies between 0,15 and $0,5 \mathrm{~Hz}$.

As we can see figure 4, the majority of the indexes of synchrony of all the subjects trials are over 0,5 proving that the simple synchrony model managed well to synchronize the robot behaviors with the blind and deaf human partners. Nerveless, in some minor trials, this model failed to synchronize NAO with the subjects. Most of the fails are logical because the subject frequency are outside the range of accepted frequencies of interaction $(0.2$ to $0.5 \mathrm{~Hz}$ ) like for the subject 2 (trial 3), subject 4 (trial 2) where the frequencies are under $0.2 \mathrm{~Hz}$ or for the subject 8 (trial 3) where the frequency is higher than $0.5 \mathrm{~Hz}$. On the other hand, for the subject 3 and 15 (moving with acceptable frequencies) the low values of the index of synchrony is due to phase shift.

\section{B. condition 2 (Unidirectional)}

The robot move with a fixed frequency of 0.35 without any possibility to change it as the camera was turned off. The subjects are asked to move here with their preferred natural frequency regardless to Nao's oscillations but with a visual focus of the arm of the robot. Consequently, the synchrony indexes show globally bad synchronizations except for the subjects 3, 5, 11 and 14 who couldn't avoid entrainment and unintentional synchronization in some of their trials (see figure 4). Nevertheless, a close look to the figure 4 representing the mean frequencies demonstrates that : (i) by comparing with the natural frequencies extracted from the condition 1, except for the subject 8 and the third trial of the subject 5, the subjects shifted their frequency toward Nao's fixed frequency.(ii) most of the shifted frequencies are very close or equal to Nao's frequency excepted for the subjects $2,3,8$ and 12 who have natural frequencies mostly under 0.25 or over $0.45 \mathrm{~Hz}$. The low synchrony index values for the other subject in due to phase shifts.

\section{C. condition 3 (Bidirectional)}

As for the condition 1, the robot is now controlled by the simple synchrony model with a turned on camera, it can consequently react and adapt its frequency relative to the human subjects movements (in a range of 0.2 to $0.5 \mathrm{~Hz}$ ). The subjects can now perceive Nao's arm. As illustrated in figure 4, we obtained globally in this bidirectional interaction enhanced 


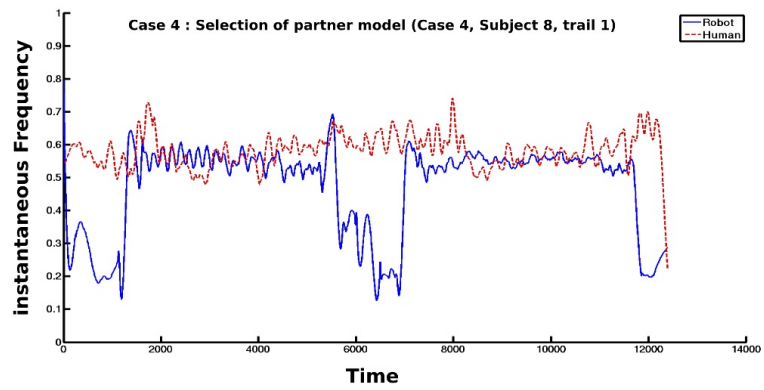

Fig. 3. Instantaneous frequencies of NAO and the subject 8 in his first trial of the condition 4

synchrony indexes if we compare with the condition 1 (blinded subjects). We can notice bad synchronization for the subject 3 (trial 2) due to his very high frequency (more than 0,5) and for the subject 12 (trial 2) and subject 15 because of phase shifts.

\section{D. condition 4 (Bidirectional)}

This condition is the most difficult to analyze because using the partner selection model, the robot behavior switches from a frequency to another every 4 seconds unless it was able to synchronize with a partner moving with frequencies inside the following ranges $[0,19$ to 0,22$] ;[0,32$ to 0,37$]$ and $[0,52$ to $0,63]$ around the three learnt frequencies $0.21,0.35$ and 0.5 .

As showed figure 4, mainly, the mean frequencies of the robot and the subjects are close but the results on synchrony indexes are mixed. This result is due to the fact that the robot behavior can be considered here as a perturbation for the natural frequencies of the subjects. Additionally using a very low coupling factor the ranges of acceptable frequencies of interaction (by NAO) are drastically reduced making it difficult to synchronize. Moreover, due to the periodic frequency switching of the robot, the mean frequencies and a global index of synchrony are not much representative of the quality of the synchronizations over time. A good example is the subject 8 (trial 1), in fact, despite a low synchrony index, if we have a close look to the figure we can notice that the subject is regularly synchronized with the highest learnt frequency of NAO.

On the other hand, when the natural frequencies of the subjects (deduced from the condition 1) are really close to the one of the three Nao's learnt frequencies, we can notice good synchronization as for the subject 2, 5 and 9 having natural frequencies respectively close to $0.2,0.35$ and 0.35 .

\section{DISCUSSION AND CONCLUSION}

Analyzing the experimental results, we can make the following deductions :

1) By comparing the condition 1 (Unidirectional) and the condition 3 (Bidirectional) that NAO and the subjects synchronize better and naturally in Bidirectional interaction. This confirmed fact was already observed for human-human interactions [46] and the necessity of minimal behavioral adaptation for social interactions.
2) From the condition 2 (Unidirectional) we can also confirm that humans could not avoid unintentional entrainment when they perceive the robot moving with a different and fixed frequency ( of $0.35 \mathrm{~Hz}$ ). However this entrainment exist but decreases if the subjects frequency is far from the robots one, from the experimental results : under 0,25 and over 0,5 .

3) The condition 3 gives the observation of easier natural and unintentional synchronization in bidirectional interaction. Moreover, an interesting fact is that mutual entrainment can be noticed as the two agents (human and robot) shift their frequency close to their partner dynamics leading to synchronizations on frequency ranges half way from the two original natural frequencies of the agents.

4) On the contrary of the condition 1 and 3, the robot in the condition 4 is highly selective. Using a very low coupling factor for this condition simulate unintentional behaviors of the robot as it can react only to partners having very close dynamics of interaction implying very low level synchronization and minimal cognitive load. The results showed effectively that humans having dynamics not close to the robot could not unintentionally synchronize. On contrary, they synchronized in the condition 3 because of the permissive coupling factor controlling the robot arm oscillator. In this third condition we can consider the robot reacting in an intentional manner.

From these global observations, we can assume that for intuitive and natural interactions, unintentional synchronization is an important parameter as it can initiate and sustain the interaction with minimal cognitive load facilitating social interaction and cognition. We proved that giving to a robot minimal abilities to adapt its behavior relative to external visual stimuli (optical flow), the human partner is able to naturally interact and coordinate with the robots dynamics through unintentional synchronization.

This mechanism (unintentional synchronization) must be important for the infant development. In fact, it can be used as a reinforcement signal to segment phase where the infant have to learn (because of the sustain of the interaction) and phases where he must stop learning as he do not have un model to imitate in order to enhance his physical and social cognition.

In short-time future works, we plan to complete this study by comparing the results with the case of intentional conditions by instructing the subjects to synchronize their movements intentionally with the robot arm movements. Additionally, we are aiming to quantify more precisely the effects of changing the coupling factors of the robots oscillators on unintentional and intentional synchronization. This coupling factor will also be measured on humans by extracting their range of "natural" frequencies of interaction.

\section{REFERENCES}

[1] M. Brass and C. Heyes, Imitation: is cognitive neuroscience solving the correspondence problem? Trends in Cognitive Sciences, no. 9, pp.489495, 2005.

[2] T. Kuriyama and Y. Kuniyoshi, Acquisition of human-robot interaction rules via imitation and response observation, in The 10th International Conference on the Simulation of Adaptive Behavior, 2008. 


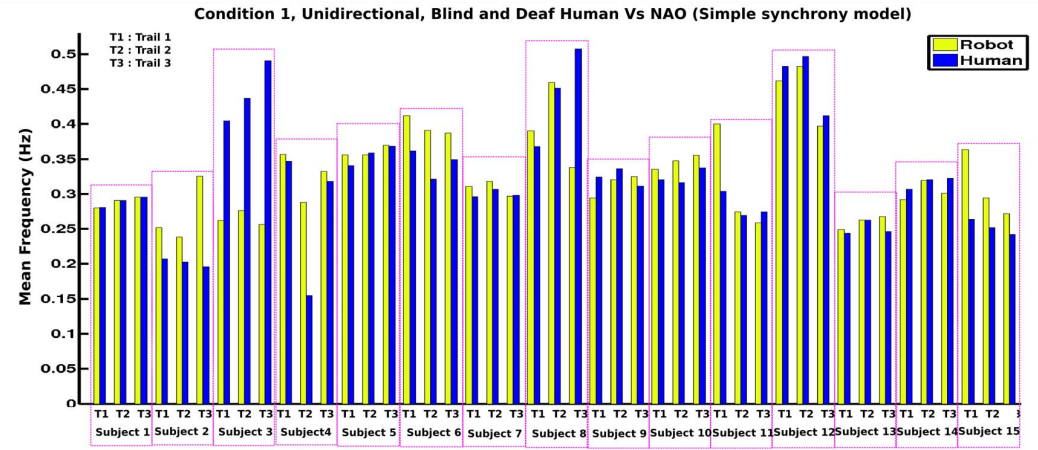

Subjects
(a)

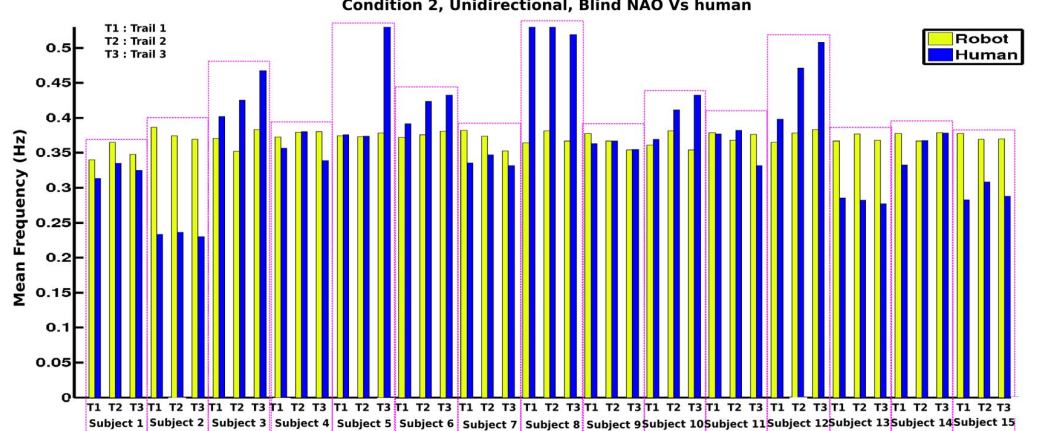

Subjects

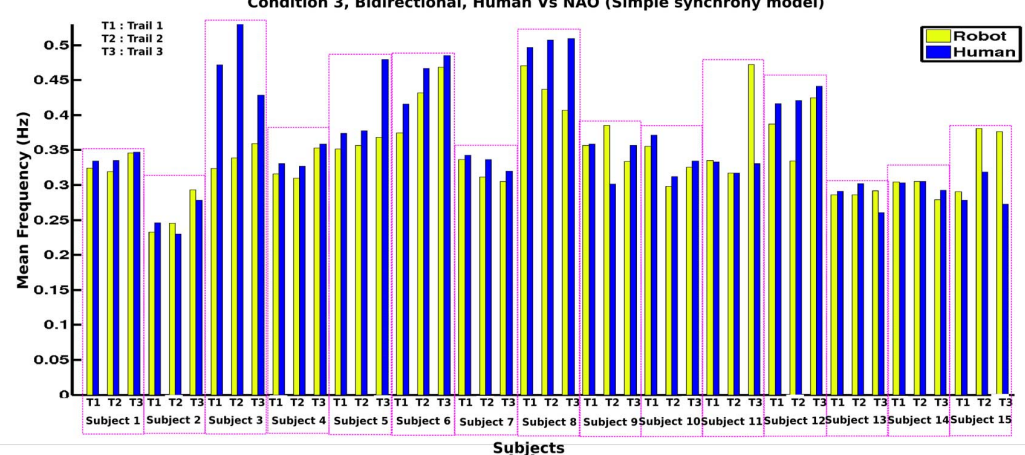

(e)

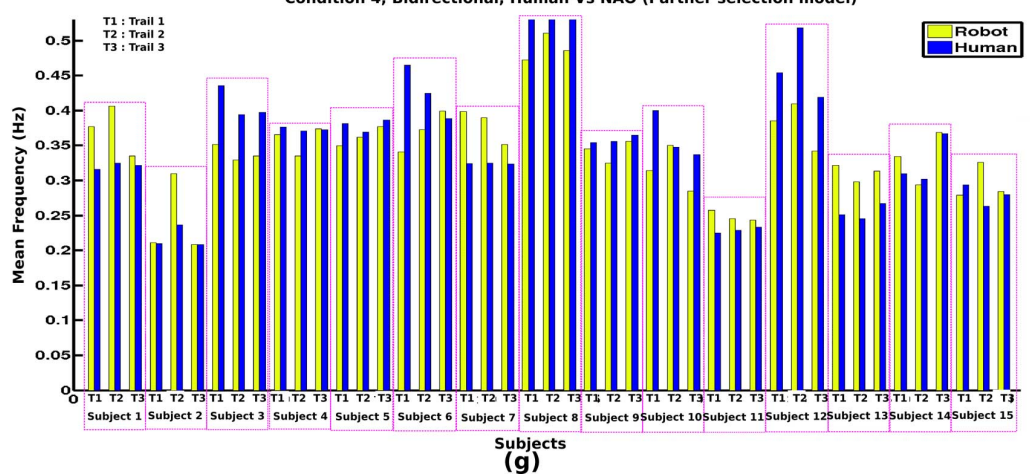

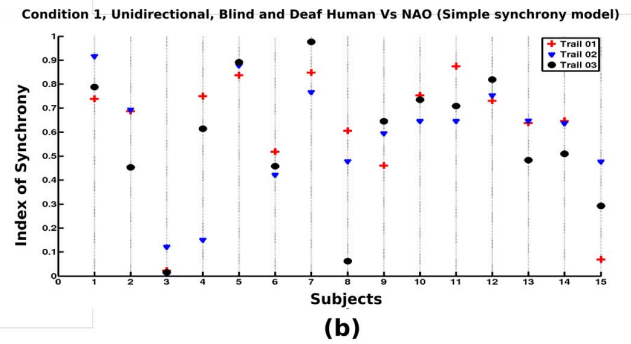

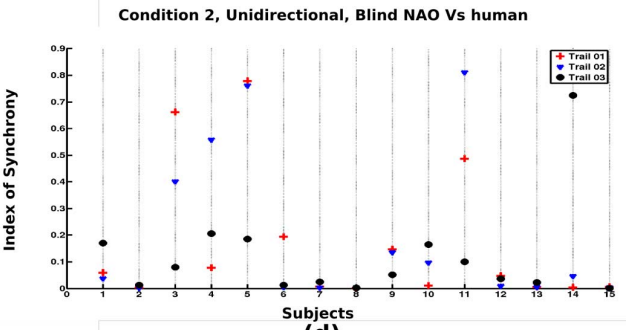

(d)
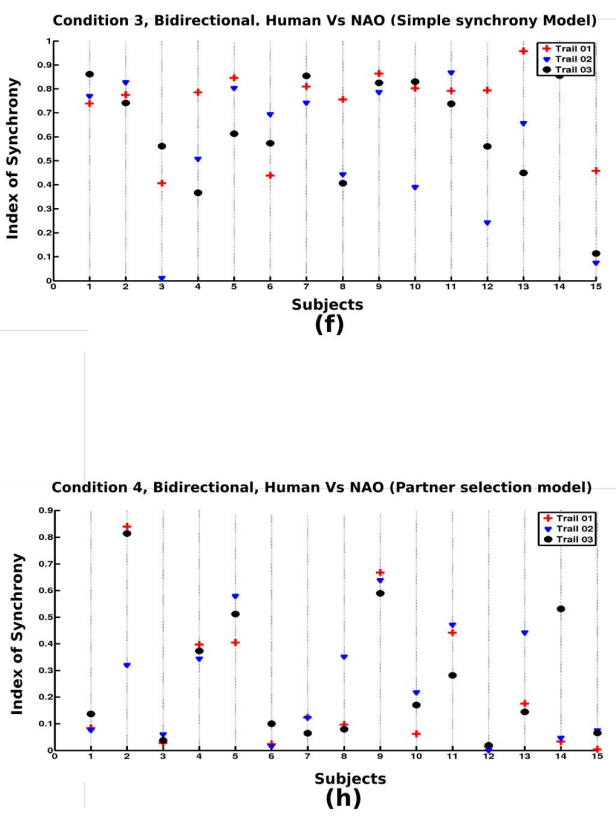

Fig. 4. Experimental results 
[3] C. Breazeal, Regulation and entrainment for human-robot interaction, International Journal of Experimental Robotics, vol. 10-11, no. 21, pp 883902, 2003.

[4] W. S. Condon. An analysis of behavioral organization. Sign Language Studies, 13:285-318, 1976.

[5] A. Kendon, R. M. Harris and M. R Key (Eds). Organization of behavior in face to face interactions. Mouton: The Hague. 1975

[6] F. Varela, Invitation aux sciences cognitives. Seuil, 1996.

[7] S. K. Hasnain, P. Gaussier, G. Mostafaoui: "Synchrony" as a way to choose an interacting partner. ICDL-EPIROB 2012

[8] R. E. Mirollo and S. H. Strogatz. Synchronization of pulse-coupled biological oscillators. SIAM Journal on Applied Mathematics 50, 1645 1662, 1990.

[9] T. J. Walker. Acoustic synchrony: two mechanisms in the snowy tree cricket. Science 166, pages 891-894, 1969.

[10] M. Rosenblum, A. Pikovsky. Synchronization: from pendulum clocks to chaotic lasers and chemical oscillators. Contemporary Physics, volume 44, number 5, pages 401-416,September October 2003.

[11] A. J. W. Ward, S. Axford, and J. Krause. Mixed-species shoaling in fish: the sensory mechanisms and costs of shoal choice. Behavioral Ecology \& Sociobiology, 52, 182187, 2002.

[12] L. J. Cator, B. J. Arthur, L. C. Harrington and R. R. Hoy. Harmonic convergence in the love songs of the dengue vector mosquito. Science, 323, 10771079, 2009

[13] L. K. Miles, L. K. Nind, and C. N. Macrae. The rhythm of rapport: Interpersonal synchrony and social perception. Journal of Experimental Social Psychology, vol. 45, 2009, pp. 585-589.

[14] R. C. Schmidt and M. J. Richardson, Coordination Neural behavioral and social dynamics (2008), Volume: socialdyna, Issue: 4, Publisher: Springer, Pages: 1-53

[15] K. Prepin and C. Pelachaud. Effect of time delays on agents' interaction dynamics. Proc. 10th Int. Conf. on Autonomous Agents and Multiagents Systems (AAMAS), Taiwan, May 2-6, 2011.

[16] G.J. Stephens, L.J. Silbert, U Hasson. Speaker-listener neural coupling underlies successful communication. Proc Natl Acad Sci USA 2010;107(32):14425:30.

[17] F. Bablioni, F. Cincotti, D. Mitta, F. De Vico Fallani, A. Tocci, L. Bianchi, et al. High resoulation hyperscanning during a card game. Conference Proceeding IEEE Eng. Med. Biol. Soc. 2007:4957-60.

[18] G. Dumas, J. Nadel, R. Soussignan, J. Martinerie, L. Garnero. Inter-Brain Synchronization during Social Interaction. PLos ONE 2010;5(8):e12166.

[19] T. Watanabe and T. Kuroda. Analysis of entrainment in face to face interaction using heart rate variablity. Proceeding of 5th IEEE workshop on Robot and Human Communication (RO-MAN6), 1414-145, 1996.

[20] H. Ogawa and T. Watanabe. InterRobot: Speech driven embodied interaction robot. Advance Robotics, 15(3), 371-377, 2001.

[21] T. Watanabe and M. Okubo. Evaluation of the entrainment between a speaker burst-pause of speech and respiration and a listener respiration in face to face communication. IEEE Workshop on Robot and Human Communication (RO-MAN7), 392-397, 1997.

[22] R. Feldman, R. M. Cohen, G. Galili, M. Singer, Y. Louzoun. Mother and infant coordinate heart rhythms through episodes of interaction synchrony. Infant Behavior \& Development 34 (2011) 569577

[23] J. Issartel, L. Marin, M. Cadopi. Unintended Interpersonal Coordination: Can we march to the beat of our own drum?. Neuroscience Letters, 411:174-179, 2007.

[24] Z. Nda, E. Ravasz, T. Vicsek, Y. Brechet, and A.-L. Barabsi, The sound of many hands clapping, Nature, 403, 850, 2000.

[25] Varlet M, Marin L, Raffard S, Schmidt RC, Capdevielle D, et al. Impairments of Social Motor Coordination in Schizophrenia. PLoS ONE 7(1): e29772. doi:10.1371/journal.pone.0029772, 2012.

[26] J. Nadel, I. Carchon, C. Kervella, D. Marcelli, D, Reserbat. Expectancies for social contingency in 2 months old. Developmental science 2, 164-173, 1999.

[27] W.S.Condon and L. W. Sander (1974). Neonate movement is synchronized with adult speech: Interactional participation and language acquisition. Science, 183, 99101.
[28] ]Meltzoff A., \& Moore M. K. (1994). Imitation, memory, and the representation of persons. Infant Behavior and Development, 17, 83?99.

[29] Chartrand, T.L., \& Bargh, J.A. (1999). The chameleon effect: The perception-behavior link and social interaction. Journal of Personality and Social Psychology, 76, 893-910.

[30] Iacoboni M. (2009) Imitation, empathy and mirror neurons. Annual Review of Psychology, 60, 653?670.

[31] Hamilton, A. F. (2008). Emulation and mimicry for social interaction: a theoretical approach to imitation in autism. Quarterly Journal of Experimental Psychology, 61, 101-15.

[32] K. Prepin and A. Revel, Human-machine interaction as a model of machine-machine interaction : how to make machines interact as humans do, Advanced Robotics. Section Focused on Imitative Robots (2), vol. 21, no. 15, pp. 1831, Dec 2007.

[33] A. Blanchard and J. Nadel, Designing a turn-taking mechanism as a balance between familiarity and novelty, in Proceedings of the Ninth International Conference on Epigenetic Robotics (EPIROB2009), November 2009, pp. 199201

[34] A. Revel and P. Andry, Emergence of structured interactions: From a theoretical model to pragmatic robotics, Neural Network, vol. 22, pp. $116125,2009$.

[35] A. Blanchard and L. Caamero. Using visual velocity detection to achieve synchronization. In Y. Demiris (Ed.), AISB05 third International Symposium on imitation in Animals and Artifacts. Hatfield UK. 2005.

[36] M. P. Michalowski, S. Sabanovic and H. Kozima. A dancing robot for rhythmic social interaction. Proceedings of the ACM/IEEE international conference on Human-robot interaction ACM New York, NY, USA 2007.

[37] T. Ikegami and H. Iizuka. Joint attention and Dynamics repertoire in Coupled Dynamical Recognizers, the proceedings of the AISB 03: the Second International Symposium on Imitation in Animals and Artifacts, pp.125-130, UK, April 2003.

[38] C. Crick , M. Munz , B. Scassellati. Robotic drumming: Synchronization in social tasks. Proceedings of IEEE International Symposium on Robot and Human Interactive Communication, 2006.

[39] V. V. Hafner and F. Kaplan. Interpersonal Maps: How to map affordances for interaction behaviors. Proceedings of international conference on Towards affordance-based robot control, 2006.

[40] S. Boucenna, P. Gaussier, L. Hafemeister, K. Bard, Autonomous Development of Social Referencing Skills. SAB 2010: 628-638

[41] Gaussier P., Baccon J.C., Prepin, K., Nadel J, Hafemeister L. (2004) Formalization of recognition, affordances and learning in isolated or interacting animats, in SAB2004, p 57-66.

[42] L. Marin, J. Issartel and T. Chaminade. Interpersonal motor coordination, from humanhuman to humanrobot interactions.In: Dautenhahn, Kerstin (ed.), Robots in the Wild: Exploring human-robot interaction in naturalistic environments: Special Issue of Interaction Studies 10:3, 2009.

[43] A. Revel, P. Andry : Emergence of Sturctured Interactions: From theoretical model to pragmatic robotics. Neural Networks, vol. 22, no. 2, pp. 116-125, 2009.

[44] K. P. B. Horn and B. G. Schunck. Determining optical flow. ARTIFICAL INTELLIGENCE, 1981, vol:17, 185-203.

[45] B. Widrow, M. E. Hoff. Adaptive switiching circuits. In IRE WESCON, pages 96-104, New York, 1960. Convention Record.

[46] Rugy A., Salesse R., Oullier O., \& Temprado JJ. (2006). A neuromechanical model for interpersonal coordination. Biological Cybernetics, 94(6), 427-443. 